\title{
DRIVING POINT IMPEDANCE CHARACTERISTICS OF THE HEAD*广
}

\author{
RICHARD L. STALNAKER and JOHN L, FOGLE \\ Biomechanics Laboratories, West V'irginia University. Morgantown. W. V. 26505. U.S.A \\ and \\ JAMES H. MCELHANEY' \\ Highway Safety Rescarch Institute. The University of Michigan. Ann Arhor. Mich. 48105. \\ L.S.A.
}

\begin{abstract}
The mechanical impedance of the human and monkey Macaca mulathal head uas determined over the frequency range $30-5000 \mathrm{~Hz}$. Miniature accelerometers and pressure transducers were placed in the brain to measure its response to vibration at constant $g$-levels and variable frequency. The maximum acceleration studied was $20 \mathrm{~g}$ s. I t vitro experiments on a fresh human cadaver and in vivo and in vitro experiments on monkeys were performed. The effect of varying blood pressure was investigated as well as the contribution to the mechanical impedance of the scalp. stull and brain.

A linear two-degree-of-freedom model that summarizes the results with acceptable accuracy is presented. Certain non-linear responses were observed for various input accelerations. No significant effect on impedance due to time after death was found for times up to five hours.

The implantable accelerometer and pressure transducer experiments indicated that the brain is very nearly critically damped. Raising the blood pressure was shown to stiffen the hrain. causing the resonance frequency of the head to increase.
\end{abstract}

\section{INTRODUCTION}

DUE To the structural and material complexity of the head. an adequate model based on continuum mechanics has not yet been developed. There are, however, several lumped parameter models available that summarize many of the observed mechanical responses of the head.

McHenry (1966) developed an 11-degreeof-freedom system which is particularly well suited for studying body kinematics and head and chest contact forces, and Martinez (1967) made use of a three-degree-of-freedom system for studying whiplash kinematics. Roberts (1969) considered the skull to be infinitely rigid and the brain mass to be translationally and torsionally connected to the skull. These models suffer from the lack of the necessary physical response data required to evaluate the many parameters of which they are composed.

The lumped model parameters such as apparent mass, damping factor, and stiffness determine the dynamic response of the model. In general. these are a function of frequency and can be evaluated by mechanical impedance techniques assuming linear system characteristics.

There has been little definitive work using impedance techniques. Franke (1956) made mechanical impedance measurements on dry human skull and on living human subjects. In these experiments the impedance transducer was held in place by a preload and was not rigidly attached to the head. This type of connection made it very difficult for the impedance transducer to remain in contact with the head near antiresonances or reson-

Receiced 7 Julv 1970.

$\div$ Presented at the 4th Annual ASME Biomechanics Technical Conference June 1-2. 1970 in Washington. D.C .

Present addren: Highway Safety Research institute. The University of Michigan. Ann Arhor. Mich. 48105. U.S.A. 
ances. A resonance of approximately $820 \mathrm{~Hz}$ was found for the dry skull and a resonance of approximately $600 \mathrm{~Hz}$ for living skulls was reported.

Hodgson (1967) has reported mechanical impedance measurements on a cadaver head. An impedance transducer was rigidly attached to the skull and loaded by a 25 -lb electromagnetic shaker. The shaker was not fastened rigidly to a large mass, but to a drill press stand. Hodgson found that the cadaver head has a resonance of $950 \mathrm{~Hz}$ with no damping. He varied the attachment point on the head and found little change in the resonance frequency. Because of the very low damping and the shaker being fastened to a rather compliant support, the results may not be completely representative of the head alone. In 1968 Hodgson ran mechanical impedance measurements on skulls filled with silicon gel, and this time the tests were carried out through a method similar to that of Franke. The results indicated an antiresonance at $313 \mathrm{~Hz}$ and a resonance at $900 \mathrm{~Hz}$ with a damping constant of approximately $2 \mathrm{lb}$-sec/in.

\section{METHODS}

The response of a linear mechanical system to a sinusoidally varying force depends on the instantaneous values of the mass, stiffness. and damping. The response may be described in terms of displacement, velocity and/or acceleration, but a description in terms of driving point impedance (the ratio of applied force to driving point velocity, (lb-sec/in.)) offers several advantages.

A body exhibiting high impedance brings to mind massiveness, stiffness, and small motion: conversely, low impedance suggests lightness. compliance and large motion. Such qualitative statements, however, are apt to be misleading. The correct view may be formed by consideration of a quantitative relationship with frequency. As the frequency with which the energy is impressed changes, both magnitude and phase of the resulting motion also change. The resistive and reactive components of impedance are complex functions of frequency and they tend to alternate in their dominance of the system response. This latter action leads to an apparent change of weight, the system responding alternately as a mass or as a spring as the frequency is varied through resonances and antiresonances. This type of response leads in turn to a great change in the degree of isolation for the components within the system and is, therefore, of importance in determining the lumped parameters of a so-called 'Black Box Model'.

If a mechanical system is harmonically excited by a force

$$
F=F_{0} \mathrm{e}^{i \omega t}
$$

which results in a velocity response

$$
V=V_{0} \mathrm{e}^{i(\omega t-\Phi)}
$$

where $\Phi$ is the phase angle between the velocity and force $w^{\text {th }}$ the force as the reference, the driving point mechanical impedance based on the force and velocity measured at the point of force application, is

$$
Z=Z_{0} \mathrm{e}^{i \phi}
$$

where

$$
Z_{0}=\frac{F_{0}}{V_{0}}
$$

There are several methods available for analytically determining the mechanical impedance. The use of the Laplace Transform is perhaps the most powerful method. An operational method developed by Firestone for the analysis of lumped-parameter systems is useful. and readily applies itself to this experiment. The mechanical impedance of living Macaca mulatta monkey and fresh human cadaver heads was determined by this method of analysis.

\section{Series element impedance experiment}

In this experiment. the monkey to be tested was anesthetized with a dose of 35 milligram 
(mg) per kilogram ( $\mathrm{kg}$ ) of sodium pentobarbital.

The force transducer was attached to the end of the hydraulic ram. A plaster of Paris impression of the right and left side of the monkey's head was made. These castings were used to support the head and to distribute the load over a wide area. The monkey's body was supported by a sling hammock attached inside a constant temperature environmental box which enclosed the entire experiment.

The plaster of Paris cast was placed on the base of the testing machine. The monkey's head was positioned on the plaster of Paris support, and the ram was lowered until contact with the temporal region of the head was made with the load transducer.

A preload of approximately $27 \mathrm{lb}$ was placed on the monkey's head. A sinusoidal force of $12.3 \mathrm{lb}$ peak-to-peak was established at a frequency of $1 \mathrm{~Hz}$. The frequency was increased by steps of ten, adjusting the displacement to re-establish a cyclic force of $12.3 \mathrm{lb}$ peak-to-peak up to $100 \mathrm{~Hz}$. This same procedure was repeated four times for each side of the monkey's head. The monkey was then sacrificed, and impedance measurements were made in the manner described above at 20 and $60 \mathrm{~min}$ after death. The body temperature was kept constant at $37^{\circ} \mathrm{C}$ by the environmental box. The skin. muscle. mandible. and brain were removed. and the driving point impedance of the skull was found by repeating the above procedure.

\section{Parallel element impedance experiment}

In this series. the Macaca mulatta monkeys were anesthetized and the right and left vertebral and common carotid arteries exposed so that they might easily be ligated subsequently. Two rectangular shaped holes were cut into the monkey's skull $\frac{3}{4}$ in. above the ear canal and $\frac{1}{2}$ in. off the sagittal suture. A combined microminiature accelerometer and pressure transducer was then implanted into the brain $\frac{1}{2}$ in. below the surface of the skull, and the dura and scalp were closed.
An X-ray was made of the head to assure alignment of the implanted transducers (Fig. 1).

The transducer was made of two dish shaped pieces of aluminum. The maximum diameter of the first dish was $0.5 \mathrm{in}$. with a base diameter of $0.312 \mathrm{in}$. and a thickness of 0.003 in. This dish was used for the pressure part of the transducer. A full bridge BLH Electronics FAES-4-18-12513 Diaphragm Gage was cemented to the base with Eastman 910. The second dish had a maximum diameter of $0.5 \mathrm{in}$. with a base diameter of $0.20 \mathrm{in}$. and a thickness of $0.035 \mathrm{in}$. This dish contained the accelerometer part of the transducer. A Clevite Corporation 56511 Piezoelectric Crystal was fastened to the base of this section. A brass weight of approximately $3.3 \times 10^{-3} \mathrm{lb}$ was then soldered to the crystal. The two halves of the transducer were then epoxyed together and waterproofed with Budd Gagekote \#4. Two such transducers were used in these experiments (Fig. 2 ).

A 10 millimeter $(\mathrm{mm})$ circular hole was then cored 0.25 in. above the ear canal on the side to be attached to the shaker. The loading fixture was then fastened to the skull at this site. On the opposite side of the skull a similar hole was made and an accelerometer was attached.

The monkey's skull via the load cell was then rigidly attached to the platen of a $200 \mathrm{lb}$ electromagnetic shaker (Fig. 3 ). The body was supported in a sling hammock, and a respirator was connected to the trachea to provide respiration when needed. A Statham Pressure Transducer was connected to the right femoral artery for monitoring blood pressure. The servo controller was then set to apply a sinusoidal constant amplitude acceleration of 10 or $20 \mathrm{~g}$ s to the head (Fig. 4). In addition. an accelerometer was placed on the free side of the head and the transmitted acceleration recorded. The implanted accelerometers were monitored through an operational amplifier and an oscilloscope. The implanted pressure transducers were also monitored through a 


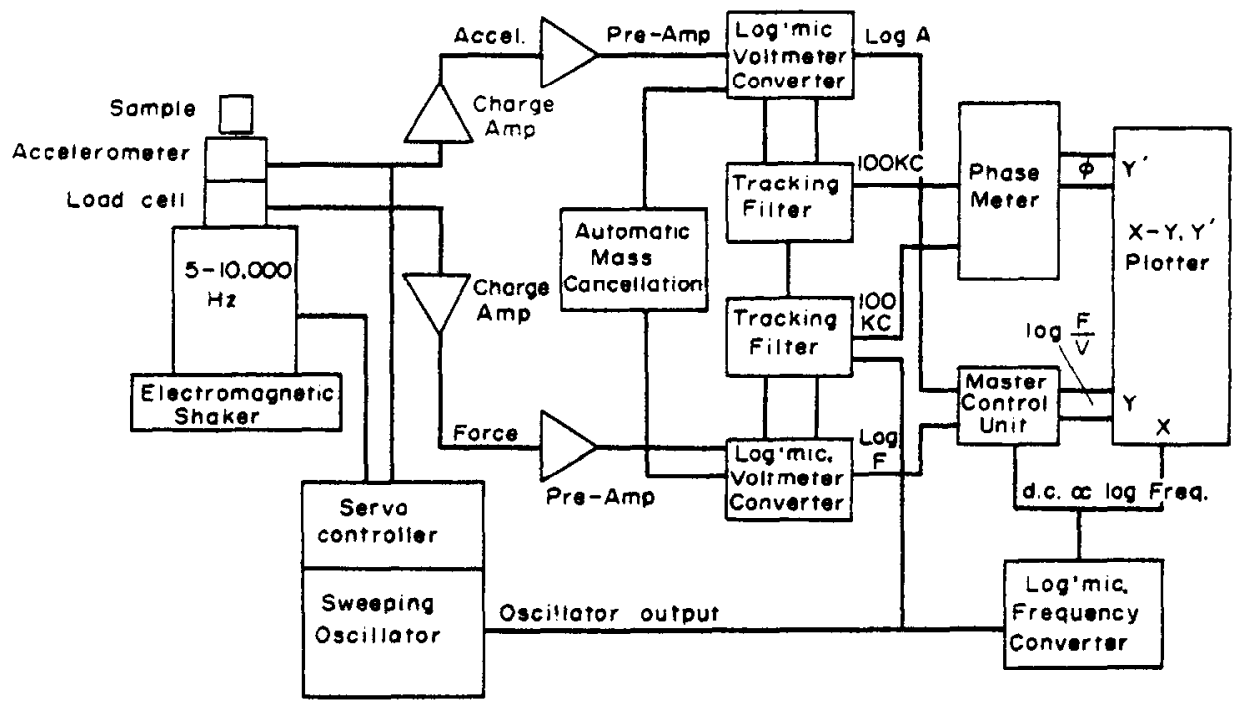

Fig. 4. Automatic impedance measuring system.

strain gage amplifier and an oscilloscope. A sweep oscillator drove the shaker system over $30-5000 \mathrm{~Hz}$. while an automatic on-line impedance computer was used to convert the force-time and acceleration-time information into a phase and impedance versus frequency plot. This impedance computer also computed acceleration ratios, pressure ratios, and associated phase angles. With this system. the test can be performed in less than 1 min (depending on sweep rate) and produces a continuous plot of impedance, pressure, and acceleration. The acceleration, the pressure in the brain. the acceleration of the skull. and the mechanical impedance were recorded for the live monkey. Then an injection of $9 \mathrm{mg} / \mathrm{kg}$ of metaraminol was given intravenously into the monkey and the above mentioned test repeated. The arteries of the monkey were then ligated for $\mathbf{3 0} \mathrm{min}$ to kill the brain and the same parameters were recorded again. The ligation was removed, allowing blood flow to resume to the head. and the above parameters were again recorded at 15 min intervals up to $3 \mathrm{hr}$ after death of the brain. The head was removed from the body and the same parameters recorded again. The skin and mandible were removed and the test repeated.
The brain was then removed through the foramen magnum and the mechanical impedance and acceleration on the free side of the skull were recorded. This procedure was followed for 5 monkeys.

The same experiment was performed on a 71 -yr old human male cadaver. This cadaver was not embalmed and had been dead approximately $30 \mathrm{hr}$ prior to the experiment. Constant accelerations of 1.5 and $10 \mathrm{~g}$ s were applied over the frequency range $30-5000 \mathrm{~Hz}$, and the above mentioned mechanical parameters measured.

In addition, three different dried human skulls were tested. The dried human skull had similar impedance characteristics to the fresh wet cadaver skull. except they showed slightly less damping.

\section{RESULTS}

\section{Series element impedance}

The mechanical impedance curve obtained from this test was a straight line of constant stiffness. Therefore. a model for this test is pure spring.

$$
Z=\frac{k}{\omega} i
$$




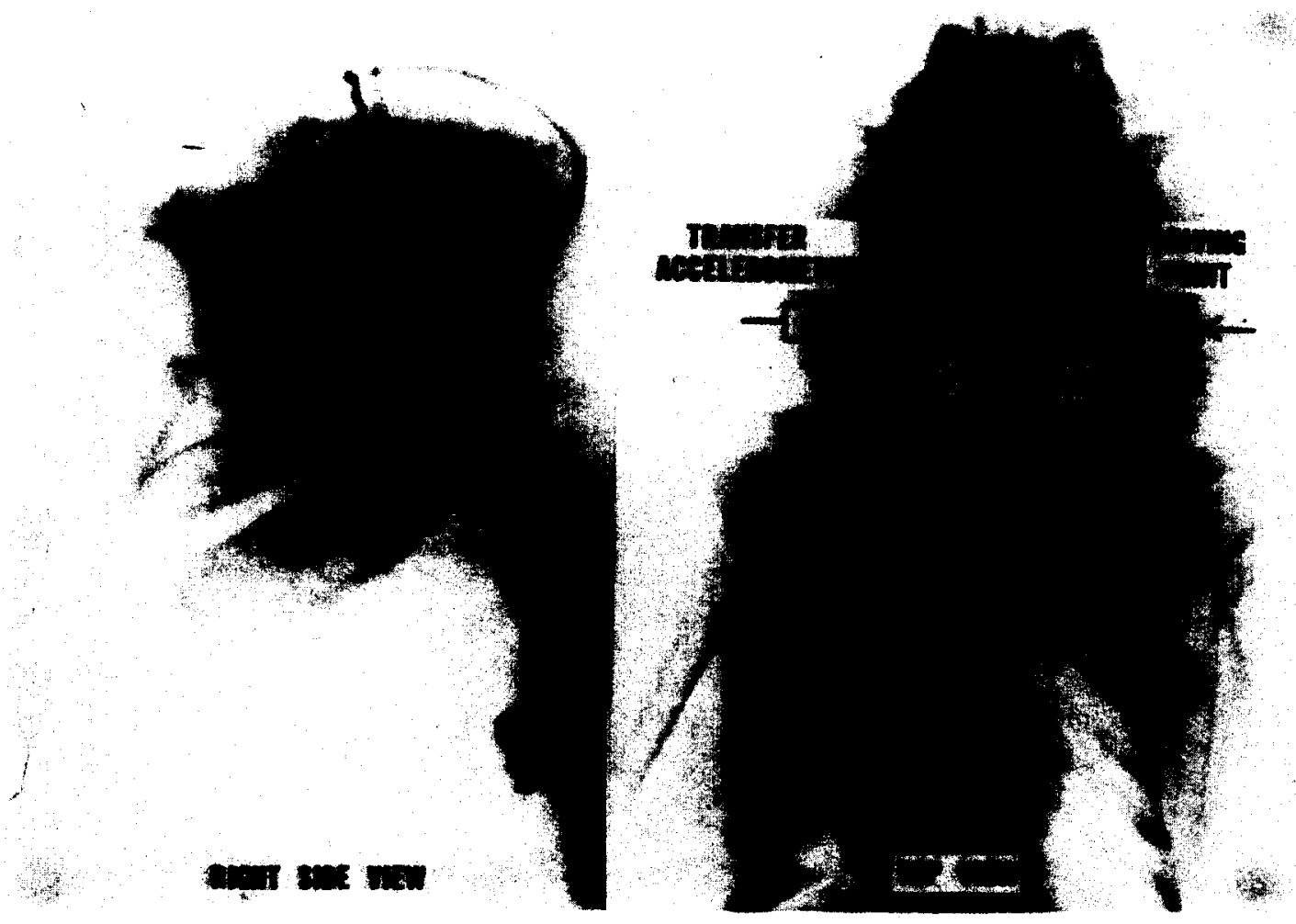

Fig. 1. X-ray of monkey with implantable transducer.

(Facing p. 130) 


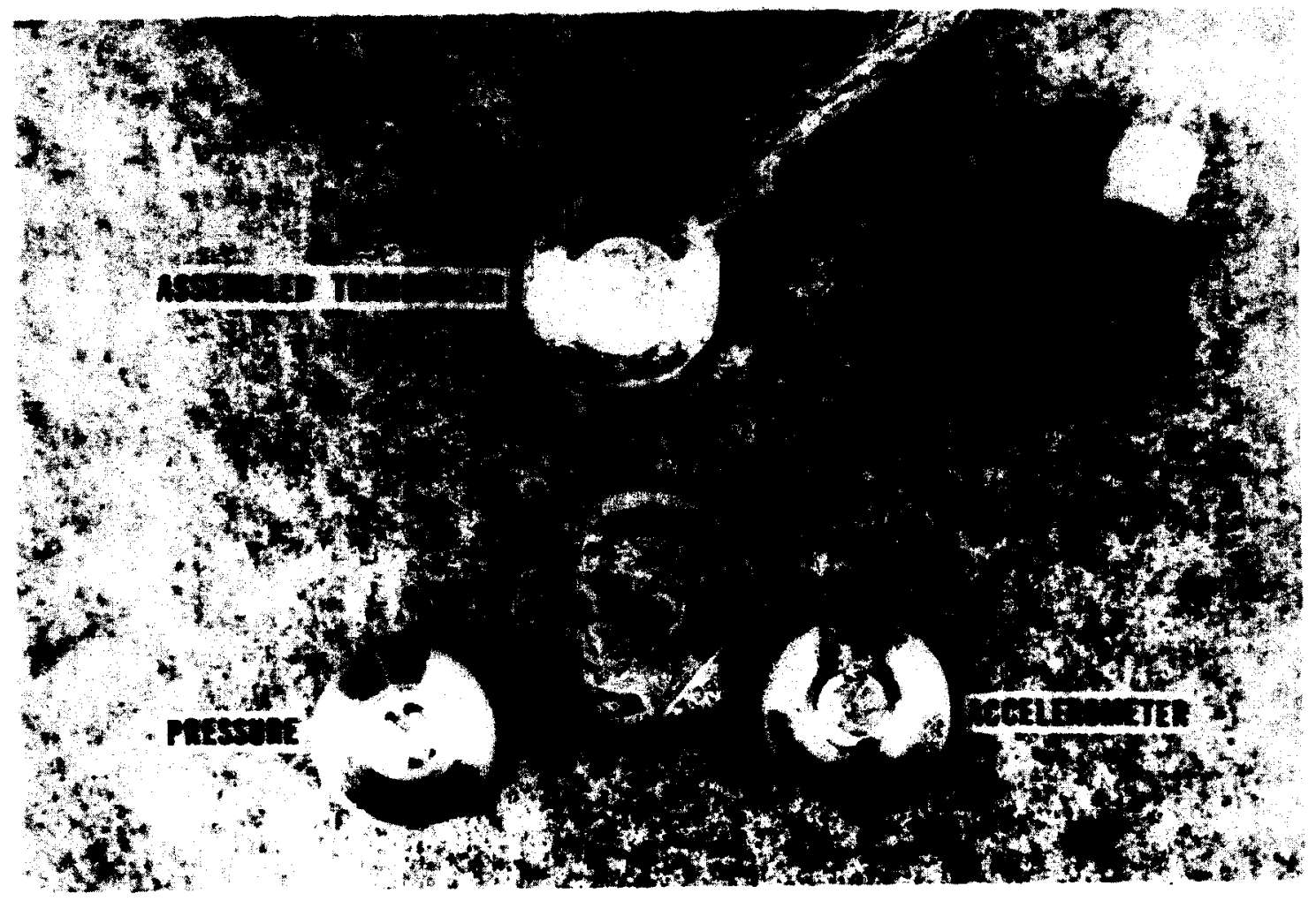

Fig. 2. Implatable accelerometer and pressure transducer. 
The spring constant ( $k$ ) for the monkey head living and dead was found to be $1.8 \times 10^{4}$ pounds per inch $(\mathrm{lb} / \mathrm{in}$.$) . When the skin and$ muscle were removed the spring constant for the skull was found to be $7 \times 10^{4} \mathrm{lb} / \mathrm{in}$. (Fig. 5).

\section{Parallel element impedance}

The mechanical impedance curves obtained from the parallel element test were analyzed by the method of Firestone. A description of this method. along with a simpie model that fits the mechanical impedance of the living monkey head will be discussed in the following paragraphs. As an example, the mechanical impedance for a spring. dash-pot, and mass will be developed. These elements will then be combined to form a simple system, and driving-point impedance expression for this system will be obtained. For harmonic motion

$$
\begin{aligned}
& \imath=i \omega x \\
& a=i \omega t .
\end{aligned}
$$

For a pure spring element. the spring constant $k$ is

$$
k=\frac{F}{x} .
$$

Substituting for $x$ in terms of velocity

$$
k=\frac{F}{l} \omega i
$$

The impedance $z_{y}$ looking into a weightless spring restrained at the opposite end is

$$
\tau_{s}=\frac{F}{i}=\frac{k}{i \omega} \text {. }
$$

Thus, spring impedance decreases as frequency increases, and the force vector leads the velocity vector by $90 \mathrm{deg}$.

The damping constant $c$ for a viscous damper is

$$
c=\frac{F}{v} \text {. }
$$

The impedance $z_{d}$ of a weightless viscous
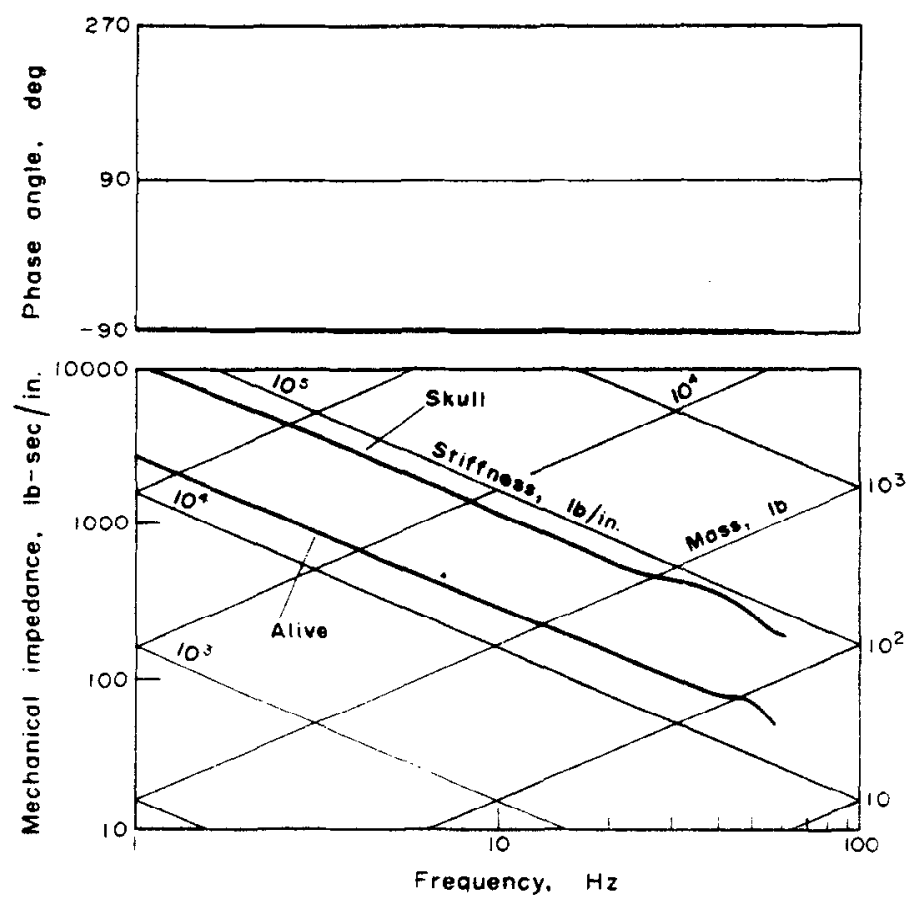

Fig. 5. Series element mechanical impedance of living monkey head and skull. 
damper attached at the opposite end is

$$
z_{d}=\frac{F}{v}=c
$$

Thus, damper impedance is independent of frequency, and force is in phase with velocity.

The impedance of a simple mass can be found from Newton's Second Law.

$$
F=m a .
$$

Substituting for $a$ in terms of $v$

$$
F=m i \omega v \text {. }
$$

The impedance $z_{m}$ of a free mass is

$$
z_{m}=\frac{F}{v}=i \omega m
$$

Therefore, mass impedance increases as frequency increases, and the force vector lags the velocity by $90 \mathrm{deg}$.

If the elements are in parallel, the elemental impedances add,

$$
z=z_{1}+z_{2}+\cdots+z_{n}
$$

But, if the elements are in series, the reciprocals of the impedance add.

$$
\frac{1}{z}=\frac{1}{z_{1}}+\frac{1}{z_{2}}+\cdots+\frac{1}{z_{n}} .
$$

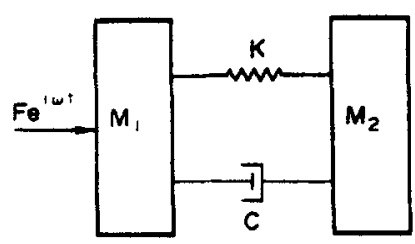

(a) Head impedonce model
The following simple model is suggested by the data obtained by using these rules.

The two-degree-of-freedom system shown in Fig. 6(a) has been developed to closely approximate the impedance characteristics of the head as measured in these experiments.

If the system is represented schematically as in Fig. 6(b), it is noted that the system elements are in both parallel and series. Using the rule of parallel systems the impedance at point 4 is

$$
z_{4}=z_{3}+z_{2}
$$

Using the rule of series system the impedance $z_{2}$ at point 2 is

$$
\frac{1}{z_{2}}=\frac{1}{z_{1}}+\frac{1}{z_{k}+z_{k}}
$$

so now

$$
z_{4}=z_{3}+\frac{1}{\frac{1}{z_{1}}+\frac{1}{z_{k}+z_{c}}}
$$

Substituting

$$
z_{4}=i \omega m_{1}+\frac{1}{\frac{1}{i \omega m_{2}}+\frac{1}{\frac{k}{\omega i}+c}}
$$

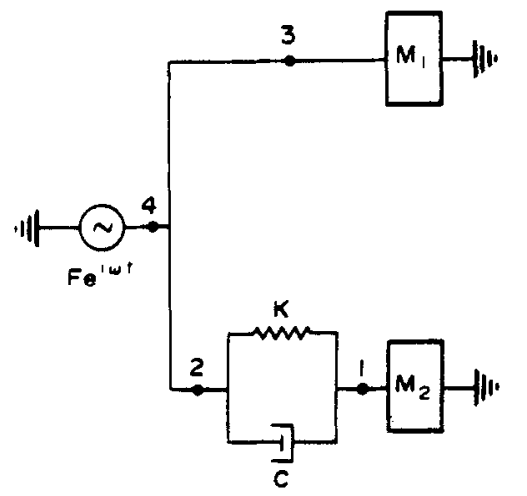

(b) Equivalent system

Fig. 6. Two-degree-of-treedom system. 


$$
z_{4}=i \omega\left(m_{1}+m_{2}\right)\left[\frac{1-\frac{\omega^{2} m_{1} m_{2}}{k\left(m_{1}+m_{2}\right)}+\frac{i \omega c}{k}}{1-\frac{\omega^{2} m_{2}}{k}+\frac{i \omega c}{k}}\right]
$$

This model has one antiresonance and one resonance. At low frequencies the system impedance approximates the total mass of the system: at high frequencies it approximates the impedance of the drive mass element $m_{1}$. The phase angle shifts from $+90^{\circ}$ through $0^{\circ}$ at the antiresonance frequency to $-90^{\circ}$, and from $-90^{\circ}$ through $0^{\circ}$ at resonant frequency back to $+90^{\circ}$. The height of the peak and the depth of the valley are controlled by the amount of damping. The spring can be approximated by the stiffness line going through the flexion point of the portion of the mechanical impedance curve between the antiresonance and resonance.

This theoretical equation was programmed for the 1130 IBM Digital Computer. By varying the magnitude of the mass, spring, and dash-pot of this model a best tit of the mechanical impedance between the model and the test data can be approximated. A plot of the model impedance and the living monkey head impedance are shown in Fig. 7. The values of the constants used in the monkey head model are $m_{1} g=0.06 \mathrm{lb}$. $m_{2} g=1.2 \mathrm{lb} . \quad k=10^{4} \mathrm{lb} / \mathrm{in}$. and $c=1.00$ lb-sec/in. With these model constants the antiresonance occurs at $270 \mathrm{~Hz}$ and the resonance at $1250 \mathrm{~Hz}$.

The range of model constants, antiresonance frequencies, and resonance frequencies for the various test configurations is given in Table 1.

The model used for the intact head is not adequate to describe the impedance characteristics of the subsections of the head. When the skin and muscle are removed a second antiresonance and resonance appears. The antiresonance and resonance become very

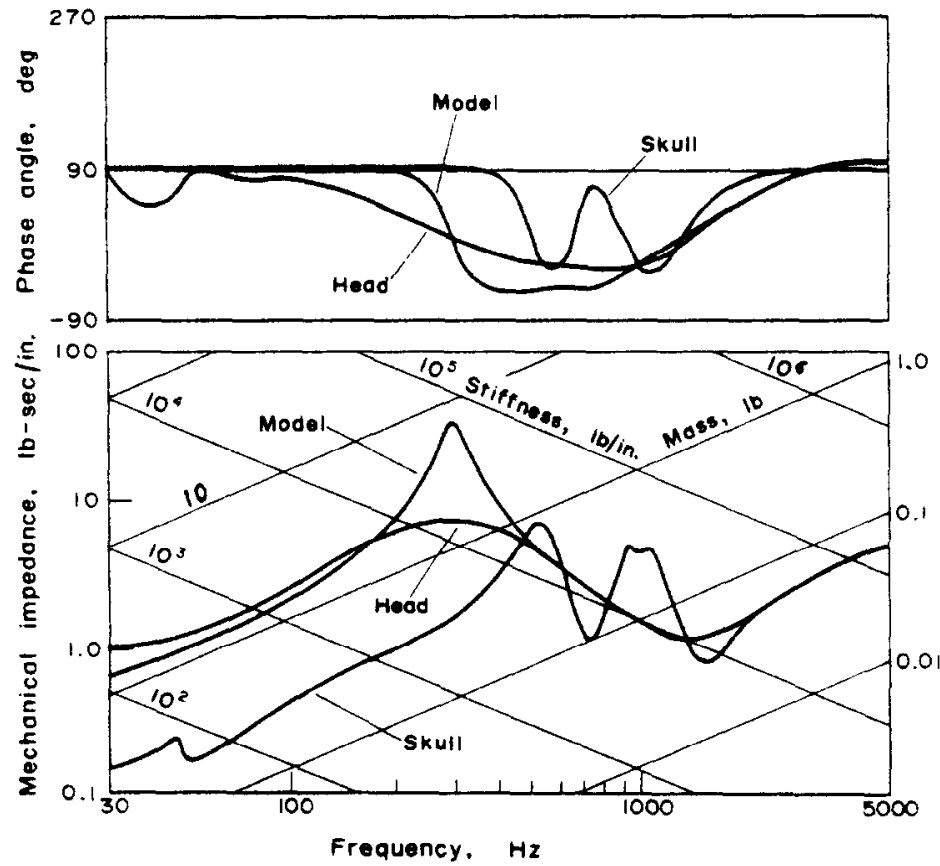

Fig. 7. Comparison of model with living monkey head and skull. 
Table 1. Range of values for assumed monkey head model

\begin{tabular}{|c|c|c|c|c|c|c|}
\hline $\begin{array}{c}\text { Test } \\
\text { configuration }\end{array}$ & $\begin{array}{r}M_{1} \\
\text { (lb) }\end{array}$ & $\begin{array}{l}M_{2} \\
\text { (lb) }\end{array}$ & $\begin{array}{c}K \\
\left(\times 10^{4} \mathrm{lb} / \mathrm{in} .\right)\end{array}$ & $\left(\begin{array}{c}C \\
\left(\frac{\mathrm{b}-\mathrm{sec}}{M}\right)\end{array}\right.$ & $\begin{array}{c}\text { Antiresonance } \\
(\mathrm{Hz})\end{array}$ & $\begin{array}{c}\text { Resonance } \\
(\mathrm{Hz})\end{array}$ \\
\hline $\begin{array}{l}\text { Alive } \\
\text { normal blood } \\
\text { pressure }\end{array}$ & $0.055-0.066$ & $1 \cdot 2-1 \cdot 7$ & $1-1.5$ & $1 \cdot 1-1 \cdot 6$ & $200-380$ & $1300-1600$ \\
\hline $\begin{array}{l}\text { Alive } \\
\text { high blood } \\
\text { pressure }\end{array}$ & $0.040-0.050$ & $1 \cdot 1-1 \cdot 2$ & $1-1.5$ & 1.0 & $300-450$ & 1600 \\
\hline $\begin{array}{l}\text { Dead } \\
\text { low blood } \\
\text { pressure }\end{array}$ & $0.050-0.060$ & $1 \cdot 0-1 \cdot 2$ & $0.8-1.3$ & $1 \cdot 0-1 \cdot 3$ & $300-480$ & 1000 \\
\hline $\begin{array}{l}\text { Head } \\
\text { body removed }\end{array}$ & $0.050-0.060$ & $0.9-1.0$ & $1-2$ & $1.0-1.7$ & 350 & $1000-1500$ \\
\hline
\end{tabular}

prominent when the brain is removed (Fig. 7). The total mass of the head, the mass being driven at high frequencies and frequencies at which the antiresonance and resonance conditions occur, can easily be estimated from the impedance frequency curves. The range of masses, resonance frequencies and antiresonance frequencies are shown in Table 2.

\section{Implanted transducer data}

Typical pressures due to sinusoidal oscillation in the living brain are given in Fig. 8. The dynamic pressure reading of the transducer AP2 closest to the shaker was found to be approximately 2.8 times larger than that of the dynamic pressure transducer API farthest from the shaker. These transducers were approximately in line with the driving

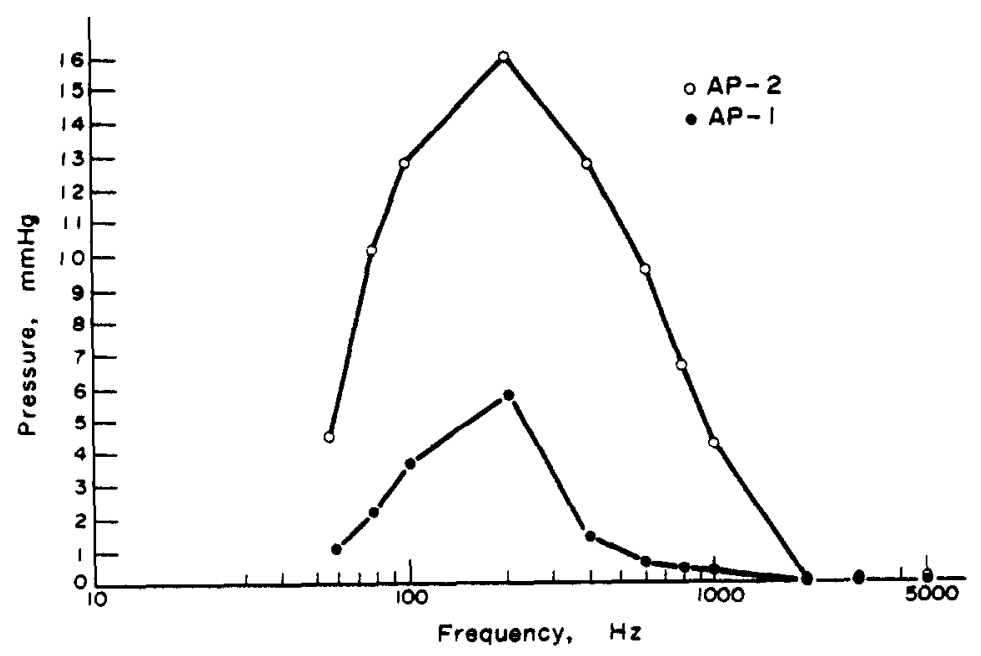

Fig. 8. Dynamic pressure in the living monkey brain. 
Table 2. Range of values for masses, antiresonances and resonances frequency of monkey skull

\begin{tabular}{lcccccccc}
\hline & $\begin{array}{c}1 \mathrm{st} \\
\text { anti- } \\
\text { resonance } \\
(\mathrm{Hz})\end{array}$ & $\begin{array}{c}1 \mathrm{st} \\
\text { resonance } \\
(\mathrm{Hz})\end{array}$ & $\begin{array}{c}\text { 2nd } \\
\text { anti- } \\
\text { resonance } \\
(\mathrm{Hz})\end{array}$ & $\begin{array}{c}\text { 2nd } \\
\text { resonance } \\
(\mathrm{Hz})\end{array}$ & $\begin{array}{c}\text { 3rd } \\
\text { anti- } \\
\text { resonance } \\
(\mathrm{Hz})\end{array}$ & $\begin{array}{c}3 \mathrm{rd} \\
\text { anti- } \\
\text { resonance } \\
(\mathrm{Hz})\end{array}$ & $\begin{array}{c}M_{1} \\
(\mathrm{lb})\end{array}$ & $\begin{array}{c}M_{2} \\
(\mathrm{bb})\end{array}$ \\
\hline Skull with brain & 300 & $550-600$ & $720-800$ & $1300-2000$ & & $0 \cdot 03-0 \cdot 05$ & $0 \cdot 5-0 \cdot 6$ \\
Skull without brain & $42-60$ & $44-80$ & $500-600$ & $600-750$ & $850-960$ & $1300-1500$ & $0.05-0 \cdot 06$ & $0 \cdot 2-0 \cdot 3$ \\
\hline
\end{tabular}

point. the $C G$ of the head and the transfer accelerometer. The distance between them was approximately one inch with AP2 being 0.75 in. from the driving point. The dynamic pressure was proportional to the blood pressure in the head. That is, with high blood pressure induced with an injection of metaraminol, the value of the dynamic pressure was highest: with the brain dead. i.e. no blood pressure, the dynamic pressure was lowest, while normal blood pressure gave an intermediate value.

A typical set of acceleration curves from an implanted transducer is shown in Fig. 9. The output from the two implanted accelerometers however. was not significantly affected by variations in blood pressure. This figure show's that over the frequency range studied in this series $(50-5000 \mathrm{~Hz})$ the accelerations in the brain are greatly attenuated.
The acceleration of the free side of the head is shown in Fig. 10. The input acceleration was a constant $10 \mathrm{~g}$ s over the entire test range of frequencies. The transmitted acceleration for the living monkey head reached a maximum of $18 \mathrm{~g}$ 's at $350 \mathrm{~Hz}$ and then fell off to $0.1 \mathrm{~g}$ 's at $2000 \mathrm{~Hz}$. The transmitted acceleration of the monkey skull alone reached a maximum of $31 \mathrm{~g} / \mathrm{s}$ at $400 \mathrm{~Hz}$ and then fell off to $0.1 \mathrm{~g}$ 's at $3000 \mathrm{~Hz}$.

The blood supply to the brain was cut off. while the rest of the monkey was kept alive. There was no change found in mechanical impedance of the monkey head for up to $5 \mathrm{hr}$ after the blood supply was cut off. When all the blood supply was cut off, changes in the mechanical impedance were noted within thirty minutes. This was due to the stiffening of the neck muscles from rigor mortis.

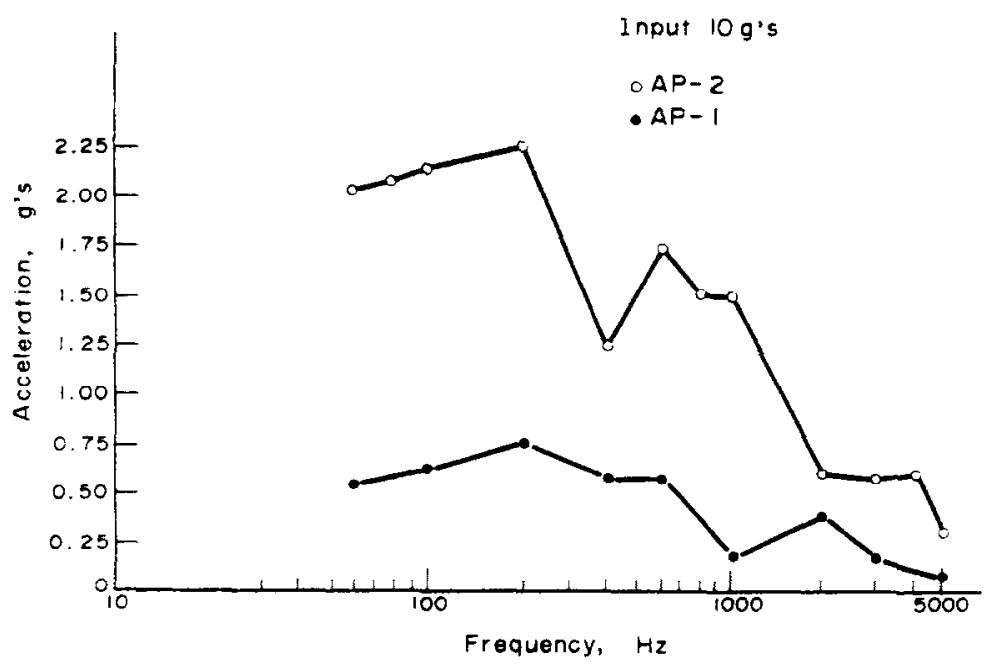

Fig. 9. Acceleration in the living monkey brain. 


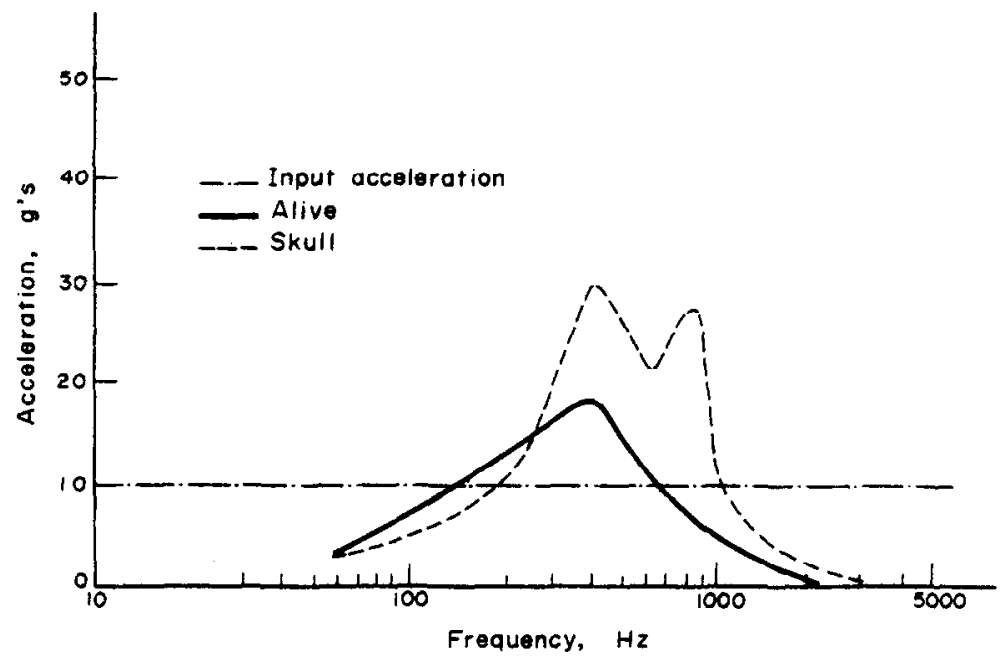

Fig. 10. Transfer acceleration of the living monkey head and skull.

\section{Human test data}

The mechanical impedance of the cadaver head was similar enough to that of the living monkey head, so the same model could be used. The theoretical and experimental mechanical impedance curve for the cadaver head are shown in Fig. 11. The model constants that provide a best fit with the cadaver head data are $m_{1} g=0.4 \mathrm{lb}, m_{2} g=9.0 \mathrm{lb}$, $c=2.4 \mathrm{lb} /$ in. $k=2.6 \times 10^{4} \mathrm{lb}-\mathrm{sec} / \mathrm{in}$, with an antiresonance at $166 \mathrm{~Hz}$ and a resonance at $820 \mathrm{~Hz}$. The human skull, however, did not


Fig. 11. Comparison of model with cadaver head and skull. 
show the same double resonance that the monkey skull showed (Fig. 11).

The linearity effects on the mechanical impedance curve of varying the input acceleration for the monkey and human skull are shown in Figs. 12 and 13. Accelerations of $1.5 .10 \mathrm{~g}$ 's for the human skull and $1,10,20 \mathrm{~g}$ 's for the monkey skull were investigated.

Variations in the mechanical impedance due to varying the input acceleration from 1 to $20 \mathrm{~g}$ 's in the case of the monkey, and 1 to $10 \mathrm{~g}$ 's in the case of the human, were not higher than 40 per cent. These variations occur between the antiresonance and the resonance frequencies. There was negligible non-linearity observed outside this region.

\section{CONCLUSIONS}

The results of these experiments indicate that the monkey and human head have approximately similar driving point impedance characteristics. The smaller mass of the monkey head, however. increased significantly the resonant frequencies. Increasing the blood pressure resulted in overall shifting effect on the impedance curve: that is, the resonant frequencies were increased approximately 30 per cent and the curves displaced to the right over the entire range.

While there were significant non-linear effects observed when the driving point acceleration was varied, the linear model developed is considered a satisfactory first approximation to the impedance characteristic of the human and monkey head when driven from the side.

The implanted accelerometer indicated that higher frequency input acceleration is greatly attenuated in the brain. thus isolating it very well from the effects of shock and vibration. The effect of the head-neck junction is small and insignificant above $400 \mathrm{~Hz}$.

The calvarium is divided into approximately four major sections: the frontal bone, left and right parietal bone, and the occipital bone. which in a monkey is almost entirely beneath the brain. The attachment to the shaker was

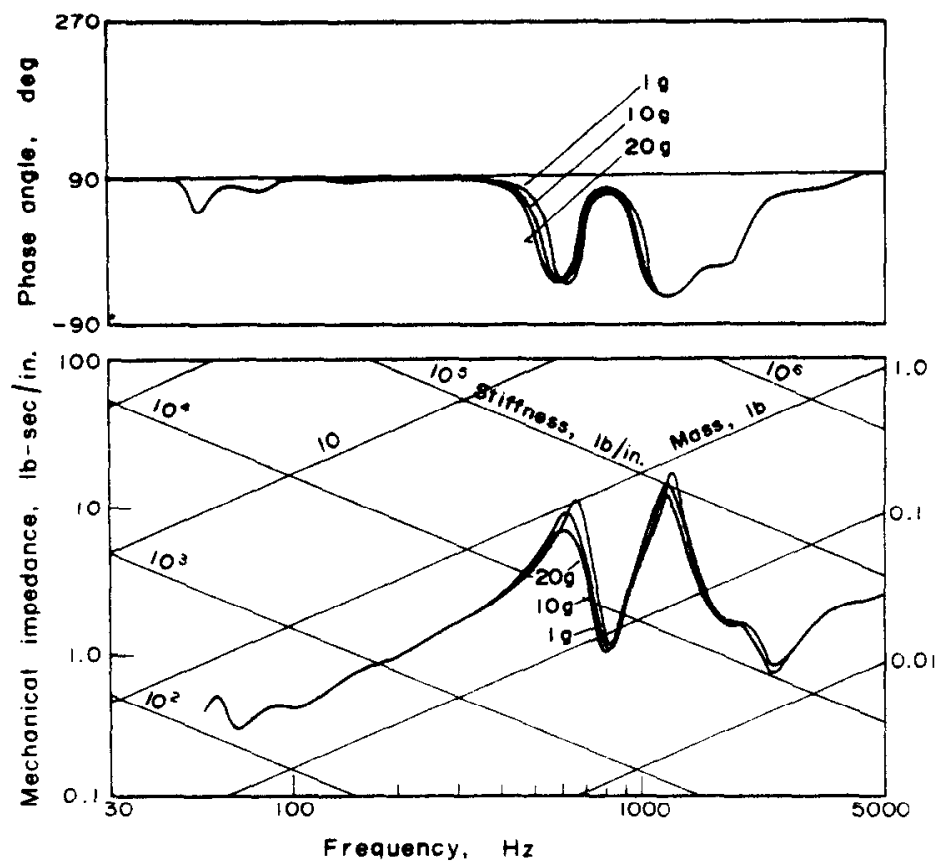

Fig. 12. Monkey skull non-linear response to acceleration. 


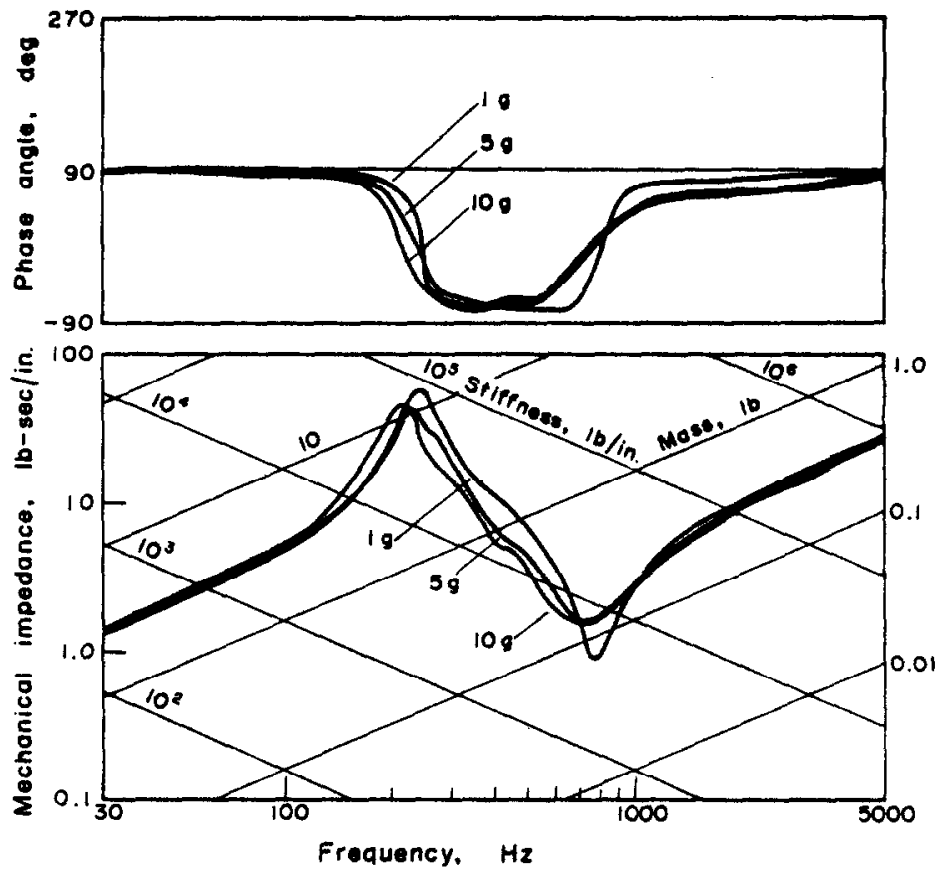

Fig. 13. Human skull non-linear response to acceleration.

made through one of the parietal sections. These sections of the skull are connected by sutures which provide isolation from one section to another. This implies that $m_{1}$ in the model may be one or more of these sections. The monkey parietal sections were found to weigh approximately $0.065 \mathrm{lb}$, thus it is believed that $m_{1}$ in the model corresponds approximately to the parietal sections of the skull. Comparing the spring constant obtained in the monkey series impedance tests $(1.8 \times$ $10^{4} \mathrm{lb} /$ in.) with the spring constant obtained through the impedance modeling $\left(10^{4} \mathrm{lb} / \mathrm{in}.\right)$ leads one to suspect that the spring element in the model corresponds for the most part to the skull stiffness. Comparing the damping constant for the whole head $(1 \cdot 2 \mathrm{lb}$-sec/in.) with that of the skull alone $(0.6 \mathrm{lb}-\mathrm{sec} / \mathrm{in}$.) indicates that the damping is due mostly to the skin, muscle and brain (Fig. 14).

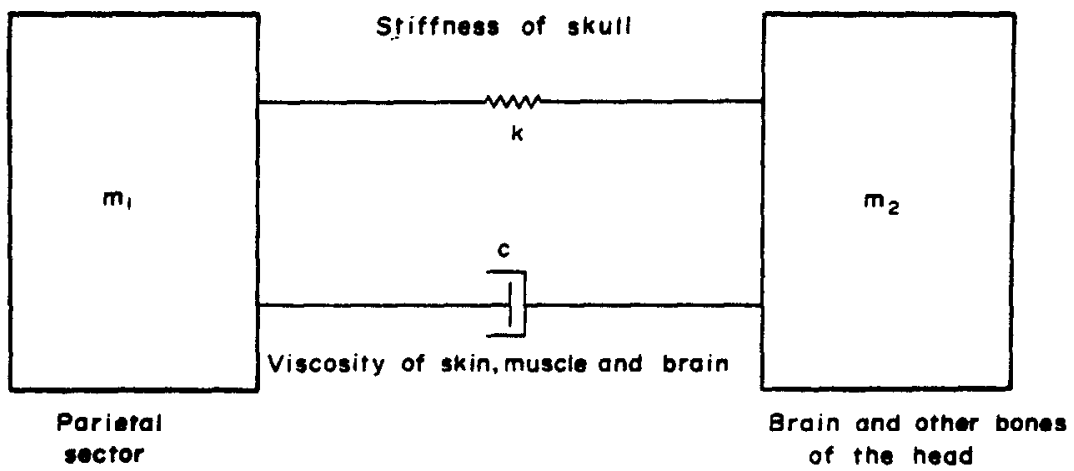

Fig. 14. Model of the head. 
Acknowledgements - The research upon which this paper is based was performed pursuant to Contract No. PH-4367-11.37 with the National Institutes of Health. Public Health Service, and Department of Health. Education and Welfare.

\section{REFERENCES}

Burstein. A. and Frankel, V. H. (1965). Viscoelasticity of biological materials. ASME Human Factors Conf., W'ash. D.C.

Chenea. P. E. (1953) On the application of the impedance method to continuous systems. J. appl. Mech.

Churcia, A. H. (1960). Mobility and impedance concepts. J. Mach. Des.

Coleman. B. D. and Noll. W. (1961) Foundations of linear viscoelasticity, Rev. mod. Phys. 32. 239-249.

Franke. E. F. (1956) Response of the human skull to mechanical vibrations. J. acoust. Soc. Am. 28, 1277.

Franke. E. K. (1951) Mechanical impedance measurements of the human body surface. USAF Tech. Rep. No. 6469 .

Frankel. U. H. and Burnstein. A. (1966) viscoelasticity of biological materials. Paper presented at Conf. at Sheletal Engng. Univ. of Florida.

Harris. C. M. and Crede. C. E. (1961) Shock Vibration Handbook. Vol. 1. Chap. 25. McGraw-Hill. New York.

Hodgson. V. R. and Patrick. L. M. (1968) Dynamic response of the human cadaver head compared to a simple mathematical model. The I2th Stapp Car Crash Conf.
Hodgson, V. R., Gurdiian、 E. S. and Thomas. L. M. (1967) The determination of response characteristics of the head with emphasis on mechanical impedance techniques. The IIth Stapp Car Crash Conf.

Martinez. J., Wickstrom. J. and Barcelo. B. (1967) The whiplash injury - a study of head-neck action and injuries in animals. Biomechanics Mono. ASME.

McHenry. R. R. and Naab. K. N. (1966) Computer simulation of the crash victim-a validation study. Proc. I2th Stapp Car Crash Conf., pp. 126-163

Roberts. S. B.. Ward. C. C. and Nahum. A. M. $11969 \mid$ Head trauma - a parametric dynamic study. ASME 69 BHF-II.

Stalnaker, R. L. (1969) Mechanical properties of the head Ph.D. Dissertation. West Virginia University.

\section{NOMENCLATURE}

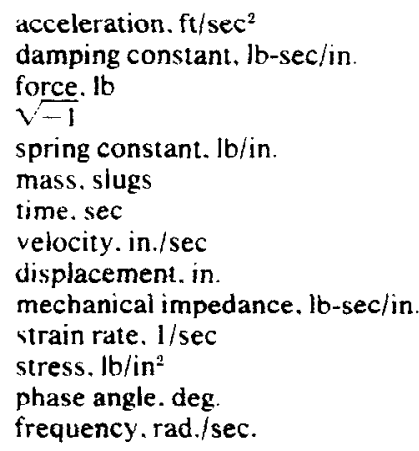

